\title{
Newborn Neurons in the Olfactory Bulb Selected for Long-Term Survival through Olfactory Learning Are Prematurely Suppressed When the Olfactory Memory Is Erased
}

\author{
Sébastien Sultan, ${ }^{\star}$ Nolwen Rey, ${ }^{\star}$ Joelle Sacquet, Nathalie Mandairon, and Anne Didier \\ Institut National de la Santé et de la Recherche Médicale (INSERM) 1028, CNRS 5292, Lyon Neuroscience Research Center, Neuroplasticity and \\ Neuropathology of Olfactory Perception Team, Université Lyon1, Villeurbanne, F-Université de Lyon, F-69007 Lyon, France
}

\begin{abstract}
A role for newborn neurons in olfactory memory has been proposed based on learning-dependent modulation of olfactory bulb neurogenesis in adults. We hypothesized that if newborn neurons support memory, then they should be suppressed by memory erasure. Using an ecological approach in mice, we showed that behaviorally breaking a previously learned odor-reward association prematurely suppressed newborn neurons selected to survive during initial learning. Furthermore, intrabulbar infusions of the caspase pan-inhibitor ZVAD (benzyloxycarbonyl-Val-Ala-Asp) during the behavioral odor-reward extinction prevented newborn neurons death and erasure of the odor-reward association. Newborn neurons thus contribute to the bulbar network plasticity underlying long-term memory.
\end{abstract}

\section{Introduction}

The mammalian olfactory bulb $(\mathrm{OB})$ is with the dentate gyrus of the hippocampus, one of the two adult brain structures undergoing permanent neurogenesis (Lledo et al., 2006). It is clear from recent literature that newborn neuron survival in the OB is modulated by learning (Alonso et al., 2006; Mandairon et al., 2006b; Mouret et al., 2008; Moreno et al., 2009; Sultan et al., 2010, 2011). However, the role of adult-born neurons in memorization and/or retention of olfactory information is still debated. Associative olfactory learning is known to increase the survival of adult-born neurons in the granule cell layer of the $\mathrm{OB}$ during their critical period of synaptic integration to the pre-existing network (Alonso et al., 2006; Mandairon et al., 2006b; Mouret et al., 2008; Sultan et al., 2010), suggesting they play a role in memory. Moreover, the long-term survival of these newborn neurons has been closely correlated with the duration of memory (Sultan et al., 2010). To analyze the necessity of newborn neurons in the process of learning and recall, several recent studies have blocked neurogenesis using various methods and looked at the functional outputs of this blockade. Two studies designed to block proliferation by infusion of the antimitotic drug AraC or irradiation (both targeting the subventricular zone), reported a deficit in the retention of an associative learning task. These findings suggested a role for newborn neurons in supporting long-term memory (Lazarini et al., 2009; Sultan et al., 2010). This conclusion was

\footnotetext{
Received July 19, 2011; accepted Aug. 12, 2011.

Author contributions: S.S., N.M., and A.D. designed research; S.S., N.R., and J.S. performed research; S.S., N.R., N.M., and A.D. analyzed data; S.S., N.M., and A.D. wrote the paper.

*S.S. and N.R. contributed equally to this work.

This work was supported by CNRS and Claude Bernard Lyon1 University.

The authors declare no competing financial interests.

Correspondence should be addressed to Anne Didier, Lyon Neuroscience Research Center, INSERM 1028, CNRS

5292, Université de Lyon, 50, Avenue Tony Garnier, F-69007 Lyon, France. E-mail: didier@olfac.univ-lyon1.fr.

DOI:10.1523/JNEUROSCI.3677-11.2011

Copyright $\odot 2011$ the authors $\quad 0270-6474 / 11 / 3114893-06 \$ 15.00 / 0$
}

reinforced by the preferential functional activation of newborn neurons upon recall of the task (Sultan et al., 2010). However, two other studies using genetic ablation of neurogenesis or AraC treatment reported no alteration in learning or long-term retention of an associative olfactory task (Imayoshi et al., 2008; BretonProvencher et al., 2009). These discrepancies could arise from differences in the learning paradigms used (Mandairon et al., 2011). To address this issue of the role of adult-born neurons in olfactory memory, we chose a new approach consisting of modulating the memory trace rather than the rate of neurogenesis. If newborn neurons support long-term memory, then erasing the memory should impair long-term survival of these newborn neurons. One ecological way of challenging this hypothesis is to create a specific memory trace associating an odor and a reward, to then behaviorally break this association, and then to check whether the newborn neurons were suppressed when the learned significance of the odor was lost.

\section{Materials and Methods \\ Animals}

Eighty male C57BLack6/J mice (Charles River) aged 8 weeks at the beginning of the experiments were used. All mice were housed under a $12 \mathrm{~h}$ light/dark cycle in an environmentally controlled room and had free access to water and food except during the olfactory learning period. All behavioral training was conducted in the afternoon (2:00 P.M. to 5:00 P.M.). The protocol was approved by the Lyon 1 ethics committee (protocol number BH2010-35), and every effort was made to minimize both the number of animals used and their suffering during the experimental procedure in accordance with the European Community Council Directive of November 24, 1986 (86/609/EEC).

\section{Behavioral experiments \\ Experimental setup}

All mice were tested on a computer-assisted two-hole board apparatus run by specific software (Mandairon et al., 2006b, 2009). The trial started by placing the mouse on the board, and the sequence of nose-poking into 
the holes was automatically recorded. A polypropylene swab was placed at the bottom of the hole, covered with bedding, and impregnated with 20 $\mu \mathrm{l}$ of pure odorant for trials involving odors.

\section{Odorants}

The odorants + limonene (purity > 97\%, Sigma-Aldrich) and + carvone (purity 96\%, Aldrich) were used.

\section{Associative learning}

Shaping. The mice were first trained to retrieve a reward (a small piece of cereal; Kellogg) by digging through the bedding. The mouse was put in the start area and allowed to dig for 2 min. Shaping was considered to be complete when a mouse could successfully retrieve a reward buried deep in the bedding.

Olfactory conditioning. During the olfactory learning experiments, mice were food deprived (to get a $10 \%$ reduction in body weight) beginning $5 \mathrm{~d}$ before the shaping session. Conditioning consisted of five sessions (one per day) of four trials ( 2 min per trial; intertrial interval: 15 $\mathrm{min})$. Each mouse was placed on the board and had to retrieve the reward that was now systematically associated with the odorant. To avoid spatial learning, this was randomly placed in one of the two holes; the other contained no odorant and no reward.

Visual conditioning. The above procedure was repeated except that the cue was no longer the odorant but a small red cap placed at the edge of the hole containing the reward.

In both visual and olfactory conditioning, randomization was controlled to avoid three trials in a row in which the reward would be placed in the same hole as well as strict alternation of the place of the reward.

Pseudo-conditioning. In the olfactory and visual pseudo-conditioned groups, the olfactory or visual cues were randomly associated with the hole containing the reward. Randomization was controlled as in conditioned animals. In addition, the configuration in which the reward would or would not be associated by chance to the cue over three trials of a session was suppressed.

Olfactory retention test. Nine days after the last olfactory conditioning session (and consequently $4 \mathrm{~d}$ post-visual conditioning) a retention test was performed, using the same conditions as during olfactory conditioning to check olfactory memorization of the original task.

Data analysis. For each trial, latency (time to find the reward) was recorded. For each behavioral session, mean latency was calculated and averaged within groups. All results are given as mean \pm SEM. Learning was assessed using ANOVA for repeated measures (Systat software, SSI), and pair comparisons were made using unilateral $t$ tests. Statistical significance was set at $p<0.05$.

\section{Intrabulbar infusions of benzyloxycarbonyl-Val-Ala-Asp}

Mice $(n=20)$ were anesthetized with cocktail injection of $50 \mathrm{mg} / \mathrm{kg}$ ketamine and $7.5 \mathrm{mg} / \mathrm{kg}$ xylazine (intraperitoneally), secured in a stereotaxic instrument (NSI), and implanted with bilateral guide cannulae (26-gauge; Plastics One) located just above both OBs at the following coordinates with respect to bregma: anteroposterior, $+5 \mathrm{~mm}$; mediolateral, $\pm 0.75 \mathrm{~mm}$; dorsoventral, $-0.7 \mathrm{~mm}$ with infusion cannulae extended $1 \mathrm{~mm}$ from the end of
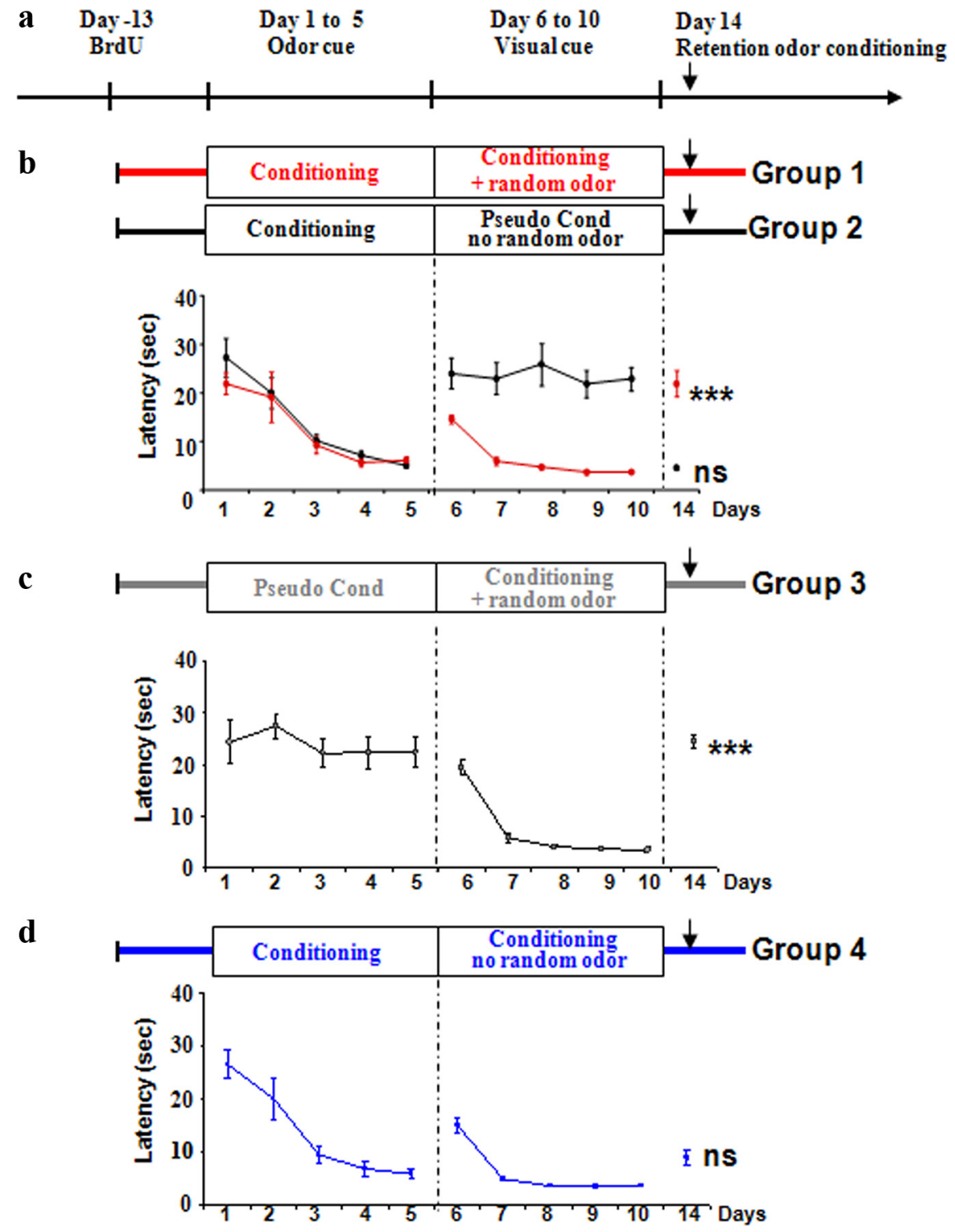

Figure 1. $\quad \boldsymbol{a}$, Timing of the experiment. BrdU was administered $13 \mathrm{~d}$ before behavioral training. Mice were trained using an olfactory cue from D1 to D5, and then using a visual cue from D6 to D10. Retention of the olfactory task was tested on D14, and the animals were killed $1 \mathrm{~h}$ after the test. $\boldsymbol{b}$, Behavioral suppression of an olfactory associative memory trace. Mice were first conditioned using an olfactory cue and then using a visual cue in the presence of the randomly presented odorant. Latency declined with sessions for olfactory $\left(F_{(4,45)}=7.86, p<0.0001\right)$ and visual learning $\left(F_{(4,45)}=51.25, p<0.0001\right)$, indicating that both were effective. Upon retention of the olfactory task, latency was higher than at D5 (D5 vs D14, $p<0.0001$ ). A control group was similarly conditioned with the odorant (decrease in latency, $F_{(4,45)}=15.36, p<0.0001$ ) but underwent pseudo-conditioning with the visual cue in the absence of any odor (no decrease in latency, $F_{(4,45)}=0.21, p=0.92$ ). Latency during the retention test was not different from the last day of olfactory conditioning (D5 vs D14, $p>0.05$ ), indicating that the olfactory task was remembered. $c$, Olfactory pseudo-conditioned mice did not learn the associative task (no decrease in latency, $F_{(4,45)}=0.54, p=0.7$ ), while the second visual conditioning task in the presence of the random odorant was acquired $\left(F_{(4,45)}=86.84, p<0.0001\right)$. Latency during the retention test was similar to the end of pseudo-conditioning (D5 vs D14, $p>0.05$ ). $\boldsymbol{d}$, Effect of visual conditioning in the absence of the random odor on retention of the olfactory task. Olfactory and visual conditioning were acquired $\left(F_{(4,40)}=1.62, p<\right.$ 0.0001 ; and $F_{(4,40)}=45.15, p<0.0001$, respectively). Latency during the retention test was similar to that on the last day of olfactory conditioning (D5 vs D14, $p>0.05$ ), indicating that the olfactory task was remembered.

the guide cannulae. Mice were allowed $10 \mathrm{~d}$ to recover from surgery in individual home cages with food and water ad libitum. BenzyloxycarbonylVal-Ala-Asp (ZVAD; $1 \mu \mathrm{g} / \mu \mathrm{l}$; in 1.5\% DMSO; Calbiochem) or saline infusions $(2 \mu \mathrm{l}$ per $\mathrm{OB})$ were performed after each visual training session.

\section{Killing}

One hour after the last behavioral trial (retention test of the olfactory association), 5 mice were randomly taken from each group, deeply anesthetized (pentobarbital, $0.2 \mathrm{ml} / 30 \mathrm{~g}$ ), and killed by intracardiac perfusion 
a

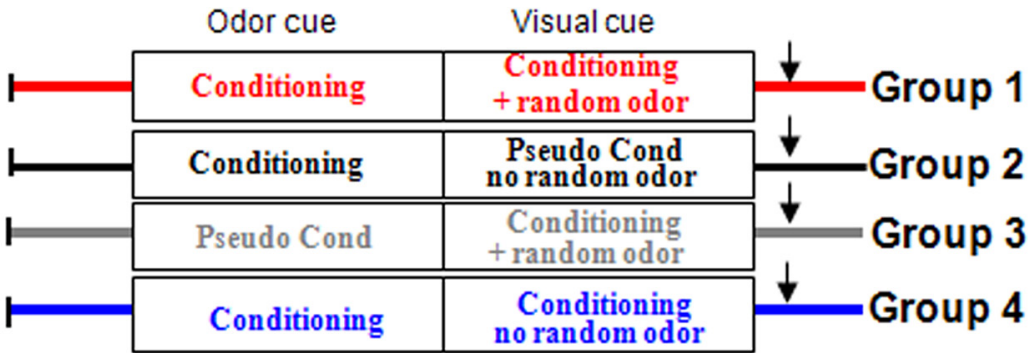

bi

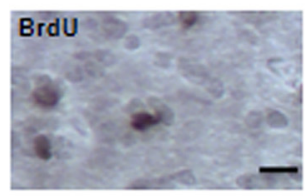

ci

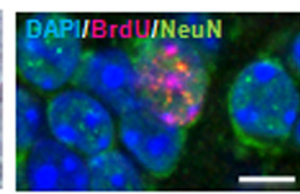

di

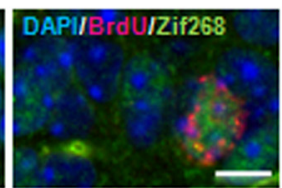

ei
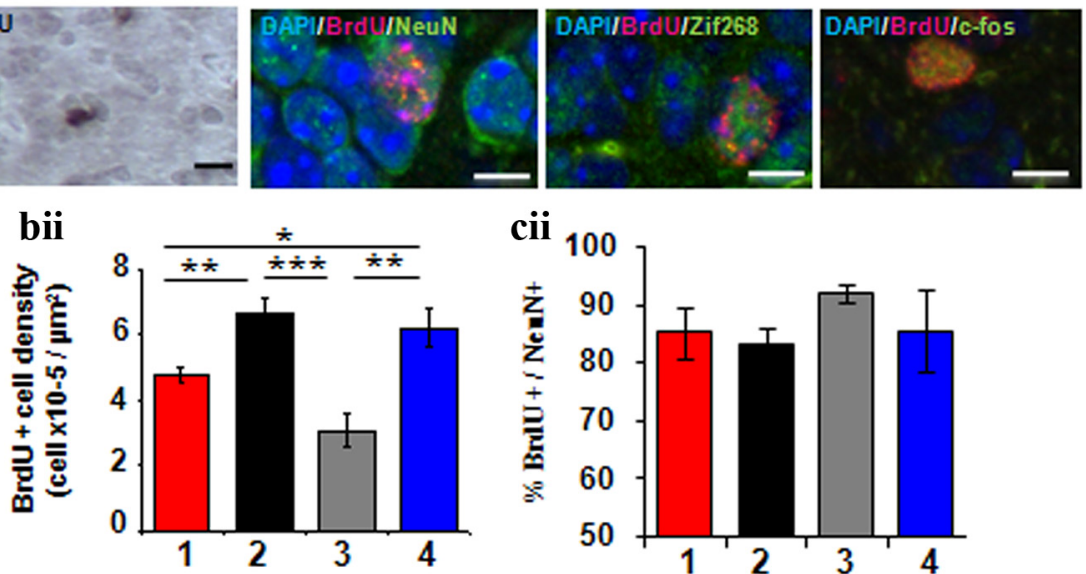

Experimental groups

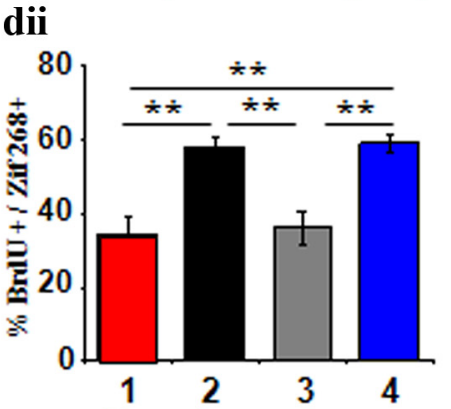

Experimental groups

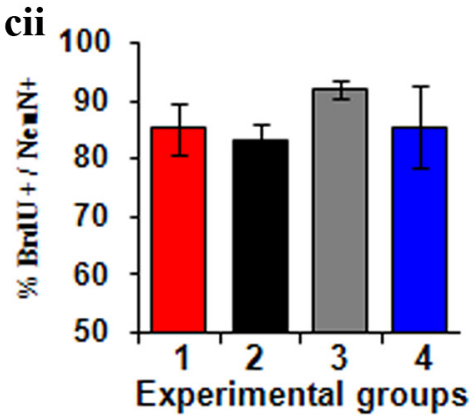

eii

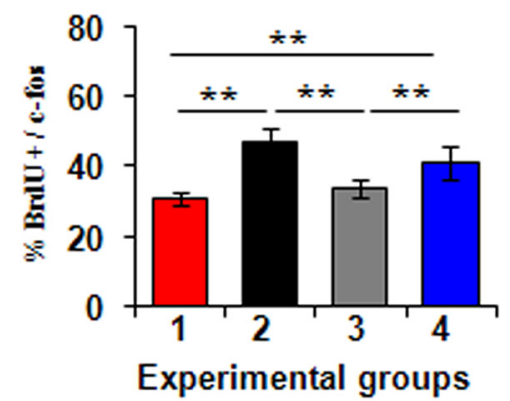

Figure 2. $\quad \boldsymbol{a}$, Experimental groups (same as in Fig. 1). $\boldsymbol{b}$, Representative BrdU-positive cell ( $\boldsymbol{b} \boldsymbol{i}$ ) and newborn (BrdU-positive) cell counts (bii) in the granule cell layer of the OB. Between-group differences were found $\left(F_{(3,14)}=12.22, p<0.0001\right)$. BrdU-positive cell density was higher in the groups remembering the associative olfactory task (groups 2 and 4) compared with the groups that forgot the task or had not learned it (groups 1 and 3). ${ }^{*} p<0.05$; ${ }^{* *} p<0.01$; ${ }^{* * *} p<0.001$. c, Neuronal differentiation of newborn cells was assessed by BrdU/NeuN double labeling (ci) and was similar in all four experimental groups (cii) $\left(F_{(3.8)}=0.74\right.$ $p=0.56)$. $\boldsymbol{d}$, Example of BrdU/Zif268 double labeling ( $\boldsymbol{d i}$ ) and double-labeled cell counts (dii) showed differential involvement of newborn neurons during the retention test $\left(F_{(3,10)}=12.2, p<0.001\right) .{ }^{*} p<0.05{ }^{* *} p<0.01 ;{ }^{* * *} p<0.001 . e$, Example of BrdU/c-fos double labeling (ei) and double-labeled cell counts (eii) showed differential involvement of newborn neurons during the retention test $\left(F_{(3,10)}=21.5, p<0.0001\right) .{ }^{*} p<0.05 ;{ }^{* * *} p<0.001$. Scale bars: bi, ci, di, ei, $5 \mu \mathrm{m}$.

of $50 \mathrm{ml}$ of $4 \%$ paraformaldehyde in phosphate buffer, $\mathrm{pH} 7.4$. Their brains were removed, postfixed, cryoprotected in sucrose (20\%), frozen rapidly, and then stored at $-20^{\circ} \mathrm{C}$ before sectioning with a cryostat (Jung).

\section{Assessment of neurogenesis}

5-Bromo-2' -deoxyuridine administration

5 -Bromo-2'-deoxyuridine (BrdU) (Sigma) $(50 \mathrm{mg} / \mathrm{kg}$ in saline, three times at $2 \mathrm{~h}$ intervals) was injected $13 \mathrm{~d}$ before the behavioral training began.

\section{BrdU immunocytochemistry}

The protocol has already been described in detail (Mandairon et al., 2006a). Brain sections were first incubated in Target Retrieval Solution (Dako) then incubated in a mouse anti-BrdU primary antibody $(1: 100$,
Millipore Bioscience Research Reagents). Sections were then incubated in a horse biotinylated anti-mouse secondary antibody (1:200, Vector Laboratories)

BrdU-positive cell quantification

All cell counts were conducted blind with regards to mouse status. Data were collected using a mapping software (Mercator Pro, Explora Nova), coupled with a Zeiss microscope. In the granule cell layer of the right $\mathrm{OB}, \mathrm{BrdU}$ positive cells were counted on 16 sections (14 $\mu \mathrm{m}$ thick, $140 \mu \mathrm{m}$ intervals, $n=5$ per group). The number of positive cells was divided by the surface of the region of interest to yield the densities of labeled cells (labeled profiles/ $\mu \mathrm{m} 2)$. Using the same method, BrdU-positive cells were counted on 7-10 sections (14 $\mu \mathrm{m}$ thick) of the dentate gyrus distributed along the anteroposterior axis. Densities were analyzed using ANOVA and unilateral Student's $t$ tests for comparison of single pairs.

\section{Double-labeling analysis}

To determine the phenotype of BrdU-positive cells in the granule cell layer of the OB, BrdU/ NeuN double labeling was performed using a rat anti-BrdU (1:100, Harlan Sera-Lab) and a mouse anti-NeuN (1:500, Millipore Bioscience Research Reagents). For functional involvement of newborn neurons, BrdU/Zif268 and $\mathrm{BrdU} / \mathrm{c}$-fos double labeling was performed using a rabbit anti-Zif268 antibody (1:1000, Santa Cruz Biotechnology) or a rabbit anti-cfos antibody (1:5000, Santa Cruz Biotechnology). The appropriate secondary antibodies, coupled to Alexa Fluor 633 to reveal BrdU and Alexa Fluor 488 (Invitrogen) to reveal other markers, were used.

BrdU-positive cells were examined for colabeling with NeuN, Zif268, or c-fos (80-100 cells per animal, $n=3-5$ animals per group). The double-labeled cells were observed and analyzed by pseudo-confocal scanning microscopy using a Zeiss microscope equipped with the ApoTome. The percentage of doublelabeled cells was calculated for each group and compared using ANOVA followed by unilateral $t$ tests.

\section{Results}

Mice were conditioned to use an olfactory cue to retrieve a food reward. This association between the food and the odor was then broken by associating the reward with a visual cue; although the odor was still present in the environment, it could no longer be used to locate the reward (Fig. 1a)

First, a group of mice (group $1, n=10$; Fig. $1 b$ ) underwent olfactory conditioning. The animals were trained over a $5 \mathrm{~d}$ period to use an olfactory cue (+limonene) to dig in the hole containing the reward. The other hole contained no odor and no reward. On the sixth day, to suppress the memory of the +limonene-reward association, the mice were similarly trained with a new task in which they had to use a visual cue, a small red cylinder placed next to the hole containing the reward. During this visual conditioning, the +limonene was randomly put in one of the two holes, and was therefore present yet had no predictive 
value for the animal (Fig. 1b). Latency (the time to find the reward) was measured as an index of performance. We found that both behavioral tasks were rapidly acquired, as evidenced by the decrease in latency (Fig. 1b). To assess whether the animals remembered the association +limonene/reward, they were tested on day 14 (D14), $9 \mathrm{~d}$ after the end of the olfactory conditioning in four trials in which the odor cue once again signaled the reward. The results of this retention test indicated that the animals did not recall the olfactory task as shown by the latency, which was higher than at the end of olfactory conditioning (D14 vs D5; Fig. 1b). Using a second cohort of animals, we tested that this memory erasure was due to the second learning and was not simply due to the time between the olfactory conditioning and the retention test. A control group (group 2, $n=10$ ) was similarly conditioned with the olfactory cue but then pseudo-conditioned with the visual cue (object randomly associated with the reinforced hole) in the absence of any odor (Fig. 1b). In this group, the olfactory task was retained as evidenced by the low latency observed during the retention test, which was similar to that of the end of olfactory conditioning (D14 vs D5; Fig. 1b). From these data, we concluded that the second conditioning was efficient in breaking the odorreward association and producing behavioral extinction of the memory trace. This paradigm was thus suitable for challenging the hypothesis that newborn neurons are suppressed with alterations to associative memory.

To assess neurogenesis, newborn neurons were counted in the granule cell layer of the $\mathrm{OB}$ using BrdU cell labeling (Fig. 1a). BrdU was injected $13 \mathrm{~d}$ before the beginning of the behavioral testing to label a cohort of newborn cells present in the $\mathrm{OB}$ at the beginning of the first task during their critical period for experience-dependent survival (Mouret et al., 2008). Importantly, we found that the BrdU-positive cell density was significantly decreased in those animals who had forgotten the olfactory association compared with the control group who had remembered it (Fig. 2bi,bii, compare groups 1,2). Double labeling of BrdU-positive cells with the neuronal marker NeuN (Fig. 2ci) indicated that newborn cells were neurons at $90 \%$ in both groups (Fig. 2cii). These results showed that breaking the odor-reward association induced a reduction in newborn neurons, strongly supporting the idea that they were indeed implicated in the memory trace and so died when the odor lost its associative value. In addition, using double labeling of BrdU-positive cells with the immediate early genes Zif268 and c-fos as indices of functional integration in the network (Magavi et al., 2005; Moreno et al., 2009; Sultan et al., 2010) (Fig. 2di,ei), we found that newborn neurons were significantly less involved in processing the learned odorant in the group that subsequently forgot it than in the group that remembered the odor-reward association (Fig. 2dii,eii, compare groups 1,2$)$. This finding suggests that the newborn neurons that disappeared from the $\mathrm{OB}$ when the task was forgotten were preferentially involved in processing the learned odor.

To further document this point, we compared neurogenesis in these first two groups with a third group of animals (group 3, $n=$ 10) that was first pseudo-conditioned with the odor cue (odorant randomly associated with the reward) and then underwent the visual conditioning with the odor cue randomly present (Fig. 1c). As expected, the animals could not learn any odor-reward association, whereas the visual cue-reward association was normally learned (Fig. 1c). We found that the level of BrdU-positive cells (coexpressing NeuN at $92.07 \pm 1.5 \%$ ) (Fig. 2cii) and the functional implication of these newborn neurons in processing the odor were lower in this group compared with the group that learned and remembered the odor-reward association (group 2),

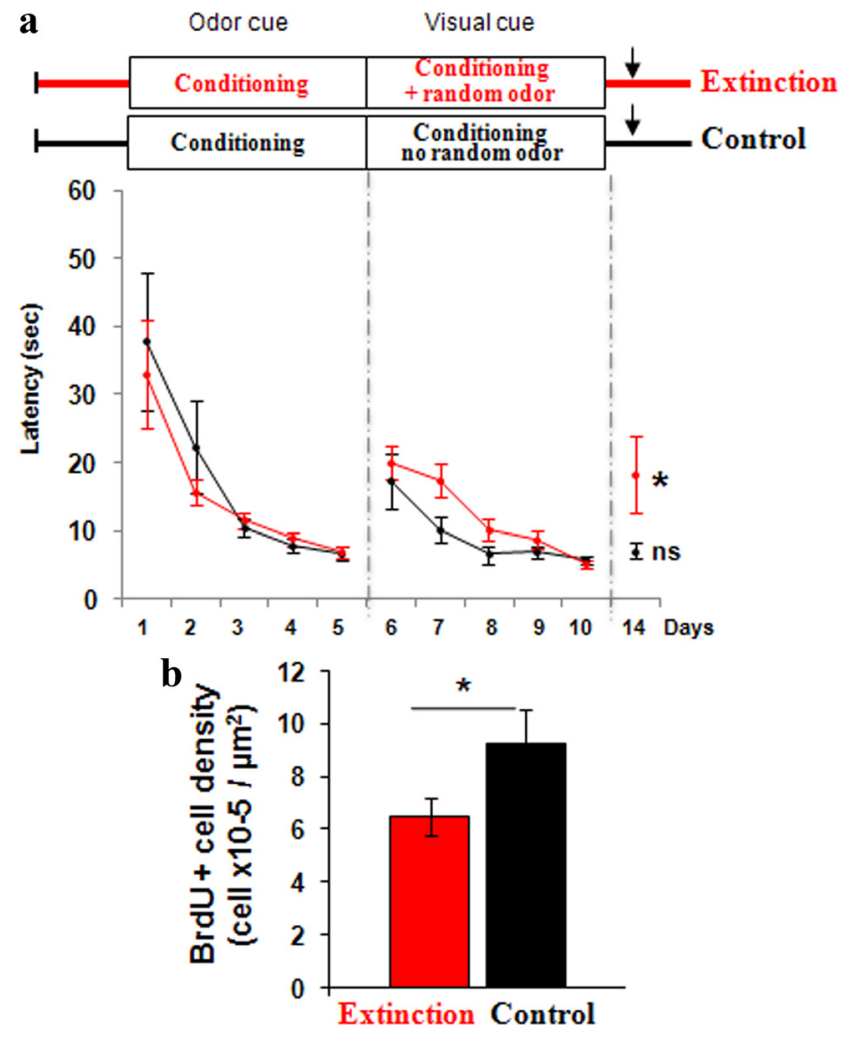

Figure 3. a, Behavioral suppression of the odor-reward association using + carvone as the olfactory cue. Mice were first submitted to the + carvone-reward conditioning then to extinction (visual cue in the presence of the random odorant) (Extinction). Latency declined with sessions for olfactory $\left(F_{(4,36)}=8.83, p<0.0001\right)$ and visual $\left(F_{(4,36)}=15.99, p<0.0001\right)$ learning, indicating that both types of learning occurred. Upon retention test of the olfactory task, latency was higher than at D5 (D14 vs D5, $p<0.05$ ). A control group was similarly conditioned but in the absence of any odor during visual conditioning (Control). Both olfactory (decrease in latency, $\left.F_{(4,32)}=7.43, p<0.0001\right)$ and visual conditioning $\left(F_{(4,32)}=6.14, p<\right.$ 0.001 ) were effective. Latency during the retention test was not different from the last day of olfactory conditioning (D14 vs D5, $p>0.05$ ), indicating that the olfactory task was remembered. $\boldsymbol{b}$, BrdU-positive cell density was higher in the Control group, which remembered the associative olfactory task, compared with the Extinction group, which forgot the task. c, BrdUpositive cell density was higher in the ZVAD-treated group, which remembered the associative task, compared with the saline group, which forgot the task. ${ }^{*} p<0.05$.

and similar to the group that learned and then forgot it (group 1) (Fig. 2dii,eii). Two conclusions can be drawn from these data. First, in line with previous studies, they indicate that learning increases the survival of newborn bulbar neurons (Alonso et al., 2006). Second, due to the BrdU injection protocol where homologous cohorts of cells were labeled in all three groups, we can conclude that newborn neurons absent in group 1 were those that failed to survive after by breaking the odor-reward association, yet had obviously been initially selected to survive during learning of the olfactory task and retrieved in group 2 (Fig. 2bii). These results thus demonstrate that breaking the odor-reward association induced a premature disappearance of newborn neurons initially selected by learning for long-term survival. These neurogenic effects were specific to the $\mathrm{OB}$ since we found no change in the level of neurogenesis in the dentate gyrus of the hippocampus (data not shown).

Finally, to determine which component of the behavioral context suppressed the odor-reward memory trace (new visual cuereward association and/or presence of the odor randomly associated with the reward), a new group of animals (group 4, $n=$ 10) first learned the odor-reward association and then the visual 


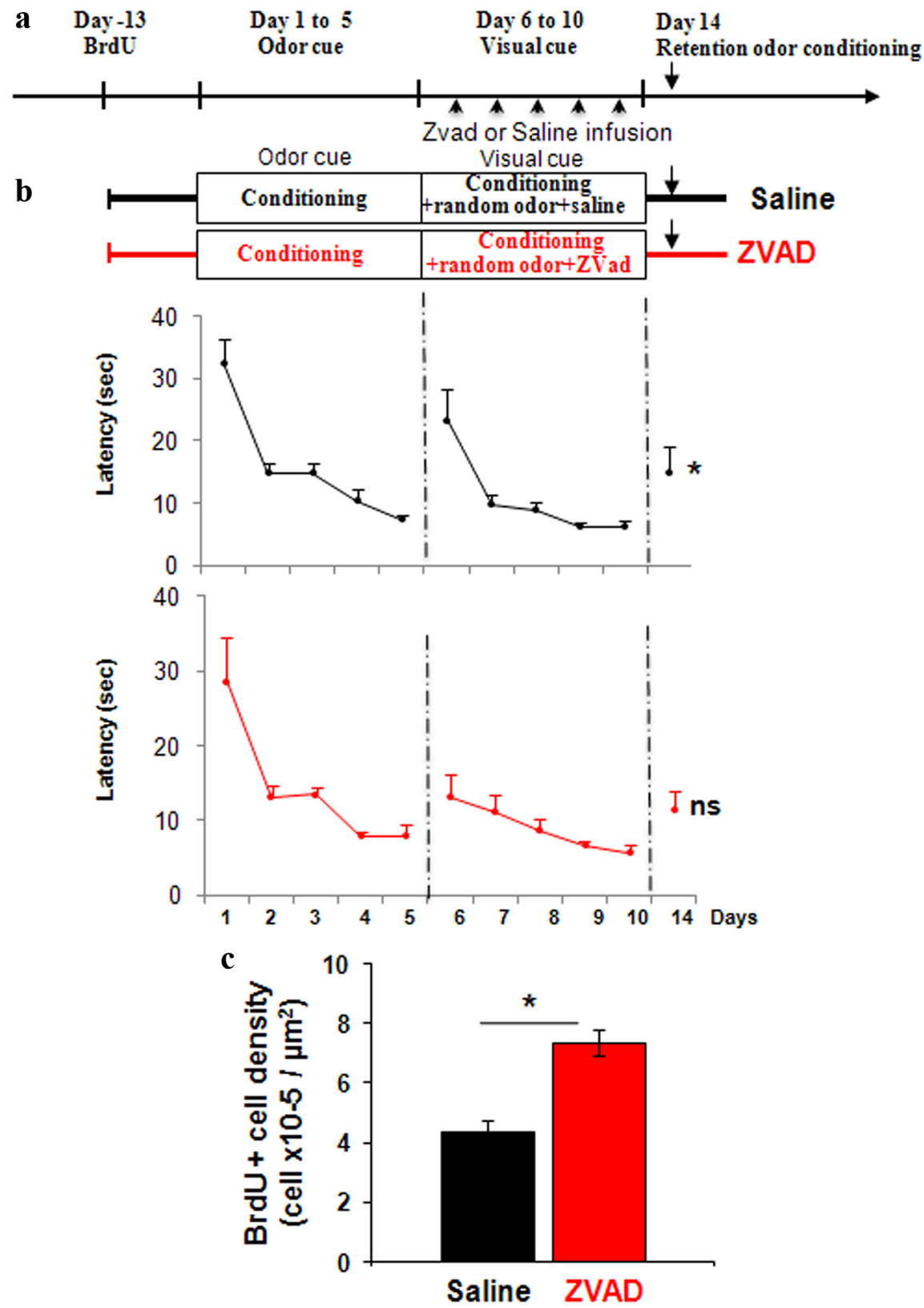

Figure 4. Behavioral suppression of the olfactory associative memory trace was prevented by ZVAD infusions in the OB. $\boldsymbol{a}$, Animals were implanted beforehand with bilateral cannulae in the $O B$, and ZVAD was infused after each session of extinction. $\boldsymbol{b}$ Behavioral data. In the two groups, olfactory (decrease in latency: Saline, $F_{(4,36)}=8.53, p<0.0001 ; Z Z A D, F_{(4,36)}=23.28, p<$ 0.0001 ) and visual (decrease in latency: Saline, $\left.F_{(4,36)}=5 ; 17, p<0.005 ; Z V A D, F_{(4,36)}=3.20, p<0.05\right)$ conditioning were effective. Latency in the retention test increased compared with the last day of olfactory training in the Saline group (D14 vs D5, $p<0.05$ ) but not in the ZVAD group (D5 vs D14, $p>0.05$ ).

cue-reward association in the absence of the odor (Fig. 1d). Retention testing showed that these animals remembered the olfactory task after the end of the second conditioning, the latency to find the reward remaining as low as at the end of the first conditioning (D14 vs D5; Fig. 1d). In line with behavior, the survival of newborn cells in this group (coexpressing NeuN at $85.18 \pm$ 4.31\%; Fig. 2cii) was higher than in the group that did not remember the task (group 1), and was similar to the group that did (group 2) (Fig. 2bii). The percentage of newborn cells expressing Zif268 or c-fos in response to the learned odorant was also similar to that of group 2 (Fig. 2dii,eii). Refusing the reliable predictive value of the odor thus allowed suppression of the associative memory trace and learning-induced neurogenesis.
To confirm these findings, we repeated the experiment of group 1 and group 4 using another odorant (+ carvone) as the olfactory cue. Two new groups of animals ( $n=10$ each) were submitted to an olfactory conditioning followed by the visual conditioning with or without + carvone being randomly associated with the reward. Similarly to what was obtained with +limonene, the group undergoing the extinction procedure could not retrieve the reward during the olfactory task as shown by the increased latency in the retention test (Fig. 3a) and showed fewer surviving newborn cells (Fig. 3b).

To confirm that newborn cells were supporting the memory of the task, we tested whether memory was retained if the death of newborn cells was inhibited during extinction. To that purpose, we infused in the $\mathrm{OB}$ the caspase pan-inhibitor ZVAD $(n=10)$ or saline $(n=10)$ after each session of extinction training in animals previously trained to the olfactory conditioning (similar to group 1), and looked at the retention of the olfactory task on D14 (Fig. 4a). Saline-infused animals showed no retention of the task, as shown by the rise in latency to find the reward, confirming results obtained in group 1 (Fig. 4b). In contrast, in ZVADtreated animals latency was not different from the last day of olfactory training, indicating that these animals remembered the odor-reward association (Fig. 4b). In parallel, we verified that ZVAD-treated OBs retained more newborn cells than saline-treated OBs (Fig. 4c). Thus, protection of the pool of newborn cells from death leads to the persistence of the memory of the task.

\section{Discussion}

Together, the present data demonstrate that newborn neurons participate in the network that processes a learned odorant. Indeed, they are removed from the network when the memory trace is no longer active, indicating that they support longterm plasticity of the neural representation of an odor and the underlying memory of its behavioral significance. Conversely, preventing the death of newborn cells allowed the memory trace to persist despite a behavioral procedure of extinction. This latter finding suggests that forgetting the odor-reward association required the disappearance of those newborn cells selected to survive during learning of the association, strengthening the conclusion that newborn cells support memory. Recent studies addressing the role of newborn neurons in olfactory memory used interventionist techniques to reduce neurogenesis yet failed to produce convergent evidence for the implication of newborn neurons in memory (Imayoshi et al., 2008; Breton-Provencher et al., 2009; Lazarini et al., 2009; Sultan et al., 2010). In the present study, 
using this new approach targeted at the behavioral alteration of memory, we have been able to demonstrate that newborn neurons selected by learning for long-term survival prematurely disappeared when the olfactory memory trace is erased. Newborn neurons in the present study were in their critical period for full integration to the bulbar network at the time of learning (Carleton et al., 2003). During this period, their survival rate is known to be influenced by learning within a few days. In close temporal correlation with the learning process, this regulation occurs through apoptotic, caspase-dependent mechanisms (Sultan et al., 2011). Our data further indicated that rapid and strong modulation of newborn cell death can also occur upon memory erasure. The cellular mechanisms underlying this postlearning regulation of cell death are also likely to involve apoptotic mechanisms since the caspase pan-inhibitor treatment was efficient in preventing death. These data strongly suggest that behavioral extinction triggered the death of newborn cells previously selected to survive, allowing in turn memory erasure.

Rapid changes in newborn neuron survival could thus underlie rapid modulation in memory strength, which could be of particular biological relevance to ensure that only current odorfood associations are retained while no longer informative cues lose their associative significance.

Together, the present findings highlight the adaptability of newborn cell survival when modulation is required to adjust the bulbar network for changes in the learned significance of an odor.

\section{References}

Alonso M, Viollet C, Gabellec MM, Meas-Yedid V, Olivo-Marin JC, Lledo PM (2006) Olfactory discrimination learning increases the survival of adult-born neurons in the olfactory bulb. J Neurosci 26:10508-10513.

Breton-Provencher V, Lemasson M, Peralta MR 3rd, Saghatelyan A (2009) Interneurons produced in adulthood are required for the normal functioning of the olfactory bulb network and for the execution of selected olfactory behaviors. J Neurosci 29:15245-15257.

Carleton A, Petreanu LT, Lansford R, Alvarez-Buylla A, Lledo PM (2003) Becoming a new neuron in the olfactory bulb. Nat Neurosci 6:507-518.
Imayoshi I, Sakamoto M, Ohtsuka T, Takao K, Miyakawa T, Yamaguchi M, Mori K, Ikeda T, Itohara S, Kageyama R (2008) Roles of continuous neurogenesis in the structural and functional integrity of the adult forebrain. Nat Neurosci 11:1153-1161.

Lazarini F, Mouthon MA, Gheusi G, de Chaumont F, Olivo-Marin JC, Lamarque S, Abrous DN, Boussin FD, Lledo PM (2009) Cellular and behavioral effects of cranial irradiation of the subventricular zone in adult mice. PLoS One 4:e7017.

Lledo PM, Alonso M, Grubb MS (2006) Adult neurogenesis and functional plasticity in neuronal circuits. Nat Rev Neurosci 7:179-193.

Magavi SS, Mitchell BD, Szentirmai O, Carter BS, Macklis JD (2005) Adultborn and preexisting olfactory granule neurons undergo distinct experience-dependent modifications of their olfactory responses in vivo. J Neurosci 25:10729-10739.

Mandairon N, Sacquet J, Jourdan F, Didier A (2006a) Long-term fate and distribution of newborn cells in the adult mouse olfactory bulb: influences of olfactory deprivation. Neuroscience 141:443-451.

Mandairon N, Sacquet J, Garcia S, Ravel N, Jourdan F, Didier A (2006b) Neurogenic correlates of an olfactory discrimination task in the adult olfactory bulb. Eur J Neurosci 24:3578-3588.

Mandairon N, Sultan S, Rey N, Kermen F, Moreno M, Busto G, Farget V, Messaoudi B, Thevenet M, Didier A (2009) A computer-assisted odorized hole-board for testing olfactory perception in mice. J Neurosci Methods 180:296-303.

Mandairon N, Sultan S, Nouvian M, Sacquet J, Didier A (2011) Involvement of newborn neurons in olfactory associative learning? The operant or non-operant component of the task makes all the difference. J Neurosci 31:12455-12460.

Moreno MM, Linster C, Escanilla O, Sacquet J, Didier A, Mandairon N (2009) Olfactory perceptual learning requires adult neurogenesis. Proc Natl Acad Sci U S A 106:17980-17985.

Mouret A, Gheusi G, Gabellec MM, de Chaumont F, Olivo-Marin JC, Lledo PM (2008) Learning and survival of newly generated neurons: when time matters. J Neurosci 28:11511-11516.

Sultan S, Mandairon N, Kermen F, Garcia S, Sacquet J, Didier A (2010) Learning-dependent neurogenesis in the olfactory bulb determines longterm olfactory memory. FASEB J 24:2355-2363.

Sultan S, Lefort JM, Sacquet J, Mandairon N, Didier A (2011) Acquisition of an olfactory associative task triggers a regionalized downregulation of adult born neuron cell death. Front Neurosci 5:52. 